\title{
APPROACHES OF NATIONAL 3D MAPPING: RESEARCH RESULTS AND STANDARDISATION IN PRACTICE
}

\author{
J.E. Stoter ${ }^{\mathrm{a}, *}$, A. Streilein ${ }^{\mathrm{b}}$, M. Pla ${ }^{\mathrm{c}}$, B. Baella ${ }^{\mathrm{c}}$, D. Capstick $^{\mathrm{d}}$, R. Home ${ }^{\mathrm{d}}$, C. Roensdorf ${ }^{\mathrm{d}}$, J.P. Lagrange \\ ${ }^{\text {a }}$ TU Delft \& Geonovum \& Kadaster, j.e.stoter@tudelft.nl \\ ${ }^{\mathrm{b}}$ Swisstopo Andre.Streilein@swisstopo.ch \\ c ICC, Catalonia,m.pla@icc.cat, b.baella@icc.cat \\ dordnance Survey, GB, dave.capstick@ordnancesurvey.co.uk, rolo.home@ordnancesurvey.co.uk, \\ Carsten.Roensdorf@ordnancesurvey.co.uk

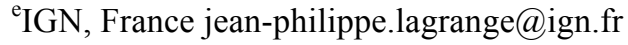

KEY WORDS: 3D national mapping; 3D standard, CityGML

\begin{abstract}
:
Over the past ten years technologies for generating, maintaining and using 3D geo-information have matured. For national mapping agencies one of the challenges is how to best extend 2D data into 3D data, making best use of research results and available technologies. Some mapping organisations are making serious progress. The question addressed in this paper is how research results achieved in the past ten years are applied in practice and what research problems remain. In addition, the paper explores the potentials of the OGC 3D standard (i.e. CityGML) for 3D national mapping and what developments are further required to make the standard better fit for this purpose. The main conclusions of the paper are that $3 \mathrm{D}$ data is more and more available but still suffers from a low level of usage (mainly visualisation) and standards and formats based on CityGML have been stabilised although software support is still in the early stage. Several recommendations are made to meet these problems, including the definition of European CityGML profiles (as the INSPIRE Building profile) to harmonise 3D needs and standardise 3D implementations at international level.
\end{abstract}

\section{INTRODUCTION}

Over the past ten years technologies for generating, maintaining and using 3D geo-information have matured. Many local governments have 3D models of their area, a large number of companies are providing services for creating or disseminating 3D models, and universities and research organisations are investigating $3 \mathrm{D}$ technologies, from capture to data services. In addition, the Open Geospatial Consortium defined the 3D standard CityGML 1.0 in 2008 and released a 2.0 version in 2012 (OGC, 2012). This has grown to a well-established standard for 3D geo-information.

CityGML originated in academia in Germany (Bonn, TU Berlin) and was originally defined as an exchange format. But it is also - and perhaps more importantly- an information model for representing 3D spatial objects. Although it started as a standard to model objects that occur in cities, the current version (2.0) has been expanded and now supports a variety of objects in landscape and city models.

Many projects use CityGML for 3D modelling. For example North-Rhine Westphalia in Germany has been the first state that provides a statewide 3D model consisting of 3D roads, railways, DTM, and 3D buildings employed for noise dispersion mapping (Czerwinski et al 2006; 2007). Another important achievement in CityGML implementation is the extension of the German national cadastre model ALKIS by 3D building models. In this project a CityGML profile has been defined and most states in Germany provide 3D building models according to this profile. In addition INSPIRE data specifications for buildings contain a $3 \mathrm{D}$ building profile in line with the CityGML specifications for buildings. Finally, different Application Domain Extensions have been developed. Some examples are noise (as used in the German state North-Rhine Westphalia and as documented within the CityGML specification), utility networks (Becker et al, 2010; 2013), real estate management, robotics, building information models (BIM), and hydrography (see CityGML, 2013).

It is clear that $3 \mathrm{D}$ applications are becoming increasingly important and therefore production of $3 \mathrm{D}$ information by governmental organisation gets more and more mandatory. For national mapping agencies, who have a long history in maintaining and incremental updating countrywide 2D-datasets, the major challenge is how to best convert this $2 \mathrm{D}$ data into $3 \mathrm{D}$ data, while supporting the established process of continuous incremental updating and making best use of research results and available technologies. Some mapping organisations are already making serious progress in this step (i.e. Swisstopo, ICC, IGN France, The Netherlands, Ordnance Survey GB).

The question addressed in this paper is how research results achieved in the past ten years in the $3 \mathrm{D}$ domain are applied in $3 \mathrm{D}$ mapping activities at national level in practice and what research problems remain. In addition, the paper explores the potential of the OGC 3D standard CityGML for 3D national mapping and what further developments are required to make the standard more appropriate for this purpose.

The paper first summarises the approaches and open issues of 3D nationwide mapping activities of the five above mentioned mapping authorities (sections 2 till 6). Then section 7 summarises the main findings, draws conclusions and identifies topics that require further (research) attention.

The material in this paper is based on the EuroSDR/OGC Workshop on "CityGML in National Mapping" that took place in January 2013, at IGN France.

The objectives of the workshop were to discuss the benefits and barriers to the provision of $3 \mathrm{D}$ geospatial data and related 
services by NMAs, explore the relationship between 2D and 3D data, identify the potentials of the CityGML standard and define requirements for future developments of the standard.

The workshop materials can be found at Geonovum (2013).

\section{3D MAPPING AT ICC: 3D DATA FOR TOPOGRAPHIC PRODUCTS}

The Institut Cartogràfic de Catalunya (ICC) is the regional mapping agency of the Government of Catalonia. From its creation in late 1982, the ICC has focused improving the cartographic production workflows.

Topographic data, ranging from scales 1:1000 to 1:25.000, are mainly collected using photogrammetric systems and stored using 2.5D data models. Elevation models are derived from these models. Smaller scales such as 1:50.000 and 1:250.000 are collected in 2D, digitized on top of orthophotoimages. Since 2002, LIDAR systems are an additional source for elevation data. A full coverage of Catalonia is available with 1 point $/ 2 \mathrm{~m} 2$ density.

ICC products, including data, metadata and specifications, are distributed free-of-charge.

\subsection{D Topographic Data in urban areas}

The topographic data set at 1:1000 scale (CT-1M) is the most detailed data covering the urban areas and the areas planned for urbanization, a total surface of 330.000 hectares. The accuracy of this product is 20 centimeters for $\mathrm{X}$ and $\mathrm{Y}$ and 25 centimeters for height. The data is compiled using photogrammetric systems, completed by data surveyed on the field, stored in an ORACLE database and distributed as geoservices and several GIS and CAD formats, including KMZ for buildings (see Figure 1). The updating cycle is between 4 and 6 years, depending on the number and importance of the changes on the territory.

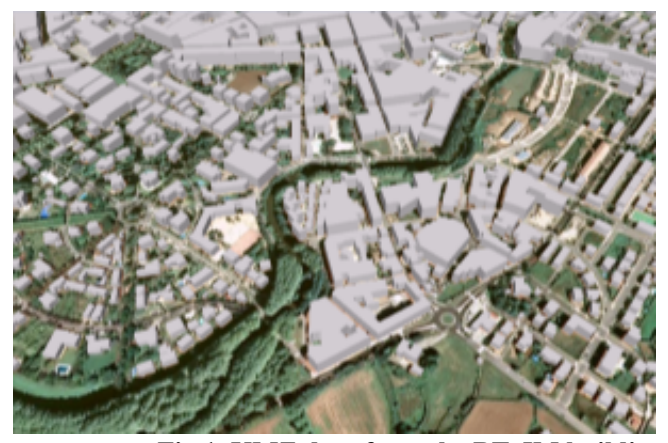

Fig 1. KMZ data from the BT-5M buildings

The first version of the data model at 1:1000 scale modelled the topographic data in 2D and used contour lines and spot heights to represent elevation. In 1999 a new data model was designed to collect data in $2.5 \mathrm{D}$, where each vertex is defined by $\mathrm{X}, \mathrm{Y}$ and $\mathrm{Z}$ coordinates. This new data model allowed new exploitations related to the three dimensionality of the information. In 2009 the third version of the data model introduced changes related to the use of the new reference system ETRS89, the adaption of the complete production process to digital technology, the adoption of standards to warrant the data and metadata interoperability and the collection of new features. The new features were added to obtain a digital terrain model (DTM) and a digital surface model (DSM), mainly used in the rectification of true orthophotos and for deriving automatically LOD1 city models (see Figure 2). All the existing data collected according to the previous data model was reused.

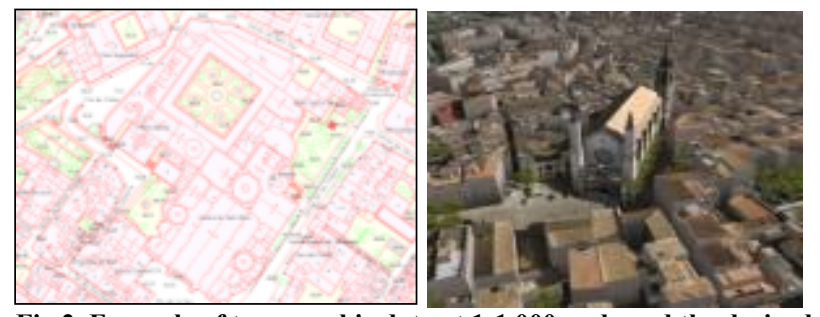

Fig 2. Example of topographic data at 1:1.000 scale and the derived city model

\subsection{The Topographic Database at 1:5000 scale}

The Topographic Database at 1:5000 scale (BT-5M) is the most detailed database that covers complete Catalonia. The data is compiled using photogrammetric systems according to a 2.5D data model and stored in an ORACLE database. The accuracy is 1 meter for $\mathrm{X}$ and $\mathrm{Y}$ and 1.5 meters for the height. During the stereoplotting process all the features required to generate a digital terrain model (DTM) and a digital surface model (DSM) are compiled together with the topographic objects (Figure 3 ). The updating cycle is 5 years over all the country and more frequently over the most dynamic areas, located mainly in the coast.

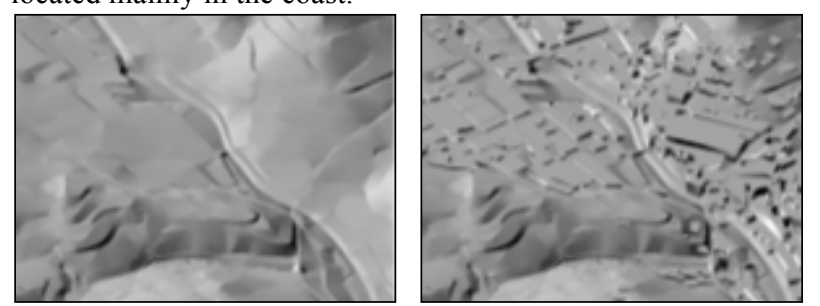

Fig 3: Elevation data from BT-5M, DTM at the left and DSM at the right

Data are distributed as geoservices and in several CAD, GIS and raster formats, including KMZ for buildings.

\subsection{The Topographic Database at 1:25.000 scale}

Although from the beginning of 2000's a complete coverage of BT-5M was available, the customers were asking for a database at smaller scale for new GIS applications and mapping. This database should be manageable, like the 1:50.000 database, but with the topographic information as detailed as possible, like the 1:5.000. Moreover, elevation data was considered essential for visualization and analysis applications. A new topographic database at 1:25.000 scale and 2.5D (BT-25M) was designed to fulfill these user requirements.

The existence of a production program for the BT-5M and the experiences at the ICC in implementing generalization workflows, allowed to start producing the first version of the BT-25M using generalization processes in the year 2003. Comparing with previous ICC generalization experiences, the workflow entailed two challenges: to obtain a topographic database, not only a map, and to generalize $2.5 \mathrm{D}$ data instead of 2D data (Figure 4). The complete coverage was finished in 2011 and, nowadays, the database is being updated independently of the original database, because the updating cycle planned for the BT-25M will be approximately 2 years, much shorter than the 5 years updating cycle of the BT-5M. 


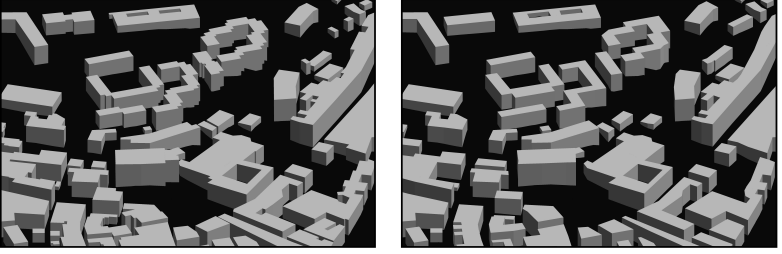

Fig 4. Example of 3D building generalization, from the BT-5M to the BT-25M

\subsection{Work in progress}

The first line of the 3D work in progress is focused in the enrichment of the CT-1M data, adding the inclined roofs for obtaining a LOD2 city models. After some tests on commercial software for obtaining inclined roofs automatically, based on image correlation and building boundaries, the results are promising although manual tasks will be required to refine them.

The second line of 3D work is the use of LIDAR data to improve the elevation data derived from the BT-5M, mainly in dense forests areas, where ground is not visible in the aerial images. Although the accuracy of LIDAR data is $0.5 \mathrm{~m}$ in $\mathrm{X}$ and $\mathrm{Y}$ and $6-10 \mathrm{~cm}$ in $\mathrm{Z}$, it must be taken into account that in cliffs and areas of high slope LIDAR data requires fine filtering. Moreover, the potential problems of incoherence between planimetric features and LIDAR data will be analyzed, for example in talwegs.

The third line of work is related to maintenance and updating of the information, crucial for optimizing the production costs. The related tasks in development are the coordination with municipalities and other government organizations to share information to speed the change detection.

Other line of work is the analysis of the compatibility between ICC data models and other models and standards, as CityGML and INSPIRE.

Finally, aspects related to dissemination of the information, are also under study. Although there is a huge potential of city model applications (urban planning, telecommunications, energy, environment, smart cities, simulations, etc.), visualization is the most common while the other ones are not yet enough developed. A 3D viewer for ICC data, considering continuous zoom using the CT-1M, BT-5M and BT-25M information, is currently in development. Also, to facilitate and spread the use of city models, an ongoing project focuses on analyzing in depth the requirements related to the use of ICC data in some specific $3 \mathrm{D}$ city model applications, in order to define some user profiles and provide data better adapted to them.

\section{3D MAPPINT AT ORDNANCE SURVEY: FROM 2D TO 3D - EXPERIENCES AND CHALLENGES}

As the National Mapping Agency for Great Britain, Ordnance Survey's products include address data, digital terrain models (DTMs), road networks, imagery and topographic mapping (vector and raster), including the flagship topographic vector mapping product, OS MasterMap ${ }^{\circledR}$ Topography Layer.

With the exception of the DTM products, Ordnance Survey's portfolio is largely 2D-centric. However, many customers are using 2D data to help make decisions that are inherently threedimensional in nature; for instance, planning and consulting on urban change, asset management, civil contingency planning and risk assessment.

This section outlines how OSGB anticipates meeting their customers' 3D needs. It will discuss issues that are being faced when moving towards a 3D-centric view. Finally, it will consider the usages and challenges being faced regarding the implementation of CityGML.

\subsection{A 3D future for Ordnance Survey?}

The long-term plan is to ' $3 \mathrm{D}$-enable' the core databases, products and services. This will involve three broad types of change (see Figure 5):

- Creating a new generation of elevation models (completed)

- Adding ground height values to 2D vertices and features in the existing 2D datasets, including topographic features and routing networks (underway)

- Generating elevations for features that extrude from the ground surface, including buildings, structures and vegetation

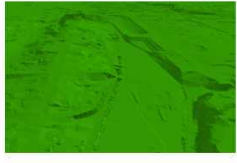

Elevation models
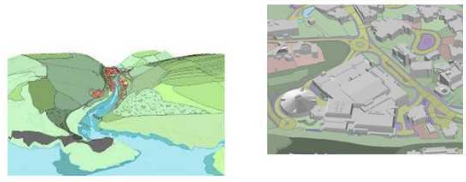

'Grounded' features 'Elevated' features
Fig 5. Categories of '3D-enabled' data

Table 1 shows the $3 \mathrm{D}$-enabled datasets that are under active consideration or development.

\begin{tabular}{|l|l|}
\hline & Datasets \\
\hline New elevation models & DTMs (complete) \\
& DSMs \\
\hline Ground heighting of existing 2D & Topographic features \\
datasets & Road network \\
& Hydrology network (complete) \\
& Rail network (complete) \\
& Tracks and Paths network \\
& Address points \\
\hline Additional capture for elevated & Building heights (LOD 1) \\
features & 3D buildings and structures (LOD2) \\
& Vegetation \\
\hline
\end{tabular}

Table 1. Existing and new products anticipated to have a 3D element

OSGB's approach to 3D capture and design is guided by a set of core principles, as outlined in Table 2 .

\begin{tabular}{|l|l|}
\hline Principle & Narrative \\
\hline Survey standards & $\begin{array}{l}\text { Data will be captured to defined standards of } \\
\text { geometric and attribute accuracy }\end{array}$ \\
\hline Maintained & $\begin{array}{l}\text { Features will be revised and updated } \\
\text { throughout their lifecycle through a } \\
\text { combination of continuous and cyclic processes }\end{array}$ \\
\hline Nationally consistent & $\begin{array}{l}\text { Equivalent geographies will (over time) be } \\
\text { published to an equivalent specification }\end{array}$ \\
\hline Intelligent & The data will enable spatial query and analysis \\
\hline Interoperable & $\begin{array}{l}\text { The data will be usable in association with both } \\
\text { Ordnance Survey and external datasets. }\end{array}$ \\
\hline Accessible & $\begin{array}{l}\text { Products will be made available in formats and } \\
\text { delivery methods which encourage adoption for } \\
\text { sustained application }\end{array}$ \\
\hline
\end{tabular}

Table 2. Core principles for Ordnance Survey 3D products

In 2012 OSGB commenced the automated capture of height values for all urban buildings and structures. Customers see this as an important evolutionary step. However, a major goal and challenge is the development of a $3 \mathrm{D}$ topography product as a 
logical upgrade from OS MasterMap Topography Layer. This product will build on the $2 \mathrm{D}$ geometry and feature identities that are already in place. Therefore the $3 \mathrm{D}$ geometry will heavily rely upon the 2D data. Initially, the focus for product generation will be on urban geographies.

\subsection{Barriers to moving from a 2D- to a 3D-centric view} OSGB considers the challenges for the step from 2D to 3D to be two-fold: technical and organisational barriers.

\subsubsection{Technical barriers}

Firstly, automated data capture techniques are preferred for minimising time and cost. This is especially relevant when scaling up to a city or national level. 3D data must also be captured during the same revision cycles as the existing 2D datasets in order to ensure synchronicity and interoperability. Since extensive use of photogrammetric techniques is being made for 2D capture, there is an obvious incentive to use similar techniques for 3D data capture to ensure this.

Secondly, data capture trials have shown that there are issues related to matching up of existing $2 \mathrm{D}$ footprints and $3 \mathrm{D}$ roof shapes. The existing 2D capture specification is oriented towards building function rather than roof form. Thought needs to be employed on how the two representations can be brought closer together. See Figure 6 for more details.

Finally, continuing investment and technical development is necessary in order to build end-to-end 3D production and supply system.

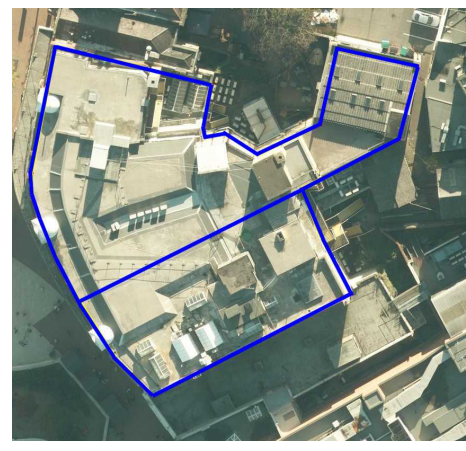

Fig 6. The 3D roof level structures clearly do not match with the 2D footprint

\subsubsection{Organisational barriers}

Ordnance Survey has for over 200 years produced 2D data. This has resulted in a certain level of learned behaviour and in a legacy of products defined in terms of 2D data specifications and representations. Consequently a process of review is necessary internally in order to gain a better understanding of $3 \mathrm{D}$ in its end-to-end sense, from capture to customer.

This 'learned behaviour' idea can also be extended to our customers. For most of OS customers 3D is a very new concept operationally. The experience of OS is that customers have a generally low level of experience and understanding of the implications and cost of using 3D data.

\subsection{CityGML for Ordnance Survey}

Feedback has shown that customers see Ordnance Survey as a prime candidate for promoting the use of $3 \mathrm{D}$ data through internationally recognised standards. CityGML fits this bill very neatly. The OGC standard CityGML is one of the few (if not only) internationally recognised standards for $3 \mathrm{D}$ geoinformation. It has a rich semantic description of terms and allows for the modelling of most of the objects found within a city. Its semantic interoperability aligned to the formal description of $3 \mathrm{D}$ features is one of its key advantages.

Ordnance Survey already supplies its detailed 2D data products in GML format, and so CityGML would seem to be a logical extension of this.

There are several ways in which OSGB could use CityGML. These include:

- As a basis for a 3D database model

- As a transfer format between internal systems e.g. from data capture to database environment.

- As a transfer format for data supply to our customers

While there are many advantages to using CityGML, OSGB also perceive some challenges, in particular:

- The need to define an accepted national profile for CityGML that is

- Fully supported by software vendors in order to exploit the richness of the information

Both of these carry the risk of resistance to the take-up of CityGML across all involved parties. Therefore the wide support of CityGML by data providers, software vendors as wells as users needs to be addressed.

\subsection{Future plans}

OSGB sees increased interest and expectation of 3D in a variety of forms from our customers. Since existing customers rely heavily on the $2 \mathrm{D}$ products, the main challenge is how to transition to a truly 3D-enabled world while maintaining continuity for customers who need support in evolving towards 3D. This also requires a balance between the growing requirement for rich $3 \mathrm{D}$ information which is more than just heighted geometry and the reluctance by customers to have to manage complexity. Above all, 3D needs to be sustainable, both in terms of production and maintenance feasibility, but also in terms of acceptability and usability by customers for solving real problems.

\section{3D MAPPING IN THE NETHERLANDS}

In The Netherlands two initiatives are on-going for nation-wide 3D mapping. The first initiative focuses on large scale data collected by local governments (mainly municipalities). The second initiative is led by the Kadaster (who also holds the mapping agencies) and aims at automatically generating a 3D version of the $1: 10 \mathrm{k}$ object oriented data set based on a smart combination of the 2D topographic data and high-resolution laser data.

\subsection{Large scale 3D mapping in The Netherlands}

The national 3D standard for large-scale topography mapping in the Netherlands was established in 2011 during the first phase of a pilot which aimed to push 3D developments in The Netherlands by collaborating with all stakeholders The Kadaster, the Ministry of Infrastructure and Environment, the Netherlands Commission for Geodesy and Geo-information and Geonovum initiated the 3D pilot (Stoter et al, 2011; Stoter et al, 2013).

The new 3D standard is embedded in an information model, called "Information Model Geography", i.e. IMGeo, which is modelled as an Application Domain Extension of the OGC 3D standard CityGML (Van den Brink 2011; 2013). IMGeo contains object definitions for large-scale representations of roads, water, land use/land cover, bridges, tunnels etc. and prescribes 2D point, curve or surface geometry for all objects. As the new version of IMGeo is modelled as CityGML ADE, it 
facilitates extensions to $2.5 \mathrm{D}$ representations (i.e. as height surfaces; equivalent to CityGML LOD0) and 3D (i.e. volumetric; i.e. CityGML LOD1, LOD2 and LOD3) representations of the objects according to geometric and semantic principles of CityGML.

Although the 3D standard is an important prerequisite for 3D applications, the implementation of the 3D standard required further agreements. Therefore, in the second phase of the 3D Pilot (run from September 2011 till December 2012) the established 3D community in The Netherlands developed readyto-use tools and best practice documents to support wide and easy implementation of the 3D standard. The deliverables of 3D Pilot NL, Phase II were published by the end of 2012 as an open source toolkit (Geonovum, 2012; partly in English). This toolkit consists of:

1. Implementation specifications for the national CityGML extension that explains all technical details of the standard, for all classes.

2. FME workbench that automatically generates $3 \mathrm{D}$ data accordingly (LOD0 for all classes as well as LOD1 and LOD2 for buildings and vegetation), based on a combination of 2D IMGeo data and high-resolution laserdata. The FME Workbench incorporates the Open Source tools developed in Oude Elberink (2010)

3. Example standard-compliant 3D data (see Figure 7) to help newcomers to understand the national 3D standard and to provide a source to experiment with 3D data.

4. 3D validator, an internationally unique, open source tool that checks 3D geometries according to ISO19107 and GML.

5. A document that describes a variety of update processes of 3D data sets based on tests with commercial software.

6. A Website that collects and portrays 3D showcases to demonstrate the added value of 3D to policy makers and newcomers in the field

7. A strategy for aligning CityGML to data generated in Building Information Models.

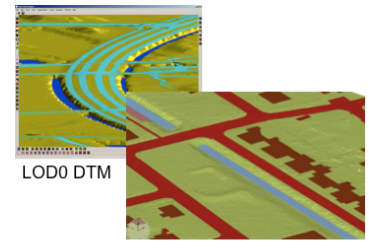

LOD2 Buildings

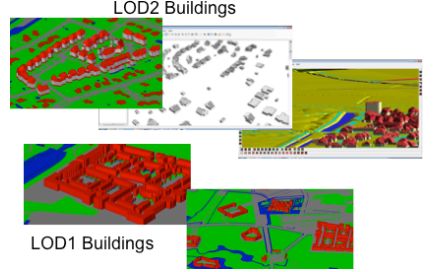

Fig. 7: 3D CityGML compliant data

\subsection{D TOP10NL}

The second initiative for national 3D mapping is the project $3 \mathrm{D}$ TOP10NL of the Dutch Kadaster (Oude Elberink et al, 2013). The project aims at automatically reconstructing a $3 \mathrm{D}$ version of the object oriented $1: 10 \mathrm{k}$ data set in a fully automatic manner and delivering this $3 \mathrm{D}$ data as open data to the community. This is done in collaboration with the universities of Twente and Delft. The automated reconstruction also uses the Open Source tools developed in Oude Elberink (2010).
Although IMGeo has been fully prepared for extension into 3D by integration of the information model with the OGC CityGML standard, 3D TOP10NL will serve other objectives. TOP10NL data is available nationwide while 2D IMGeo data will only be generated in the coming years. In addition, TOP10NL is less detailed than IMGeo and therefore better suitable for fully automatic 3D object reconstruction since fewer details are present (therefore less special cases). We assume that the resulting dataset is sufficient for a nationwide dataset (i.e. acceptable performance for nationwide applications), and that it can be further refined (for example with 3D IMGeo data) when applied in future projects.

Some tests on generating 3D TOP10NL fully automatically, have been done in the past years, see Figure 8 (Oude Elberink et al, 2012). Currently, the research results will be made further ready for practice. The developments of 3D TOP10NL builds on the insights obtained by the pilots on 3D IMGeo, since the techniques to obtain LOD0 and LOD1 representations from a combination of $2 \mathrm{D}$ data and high-resolution height points are similar.
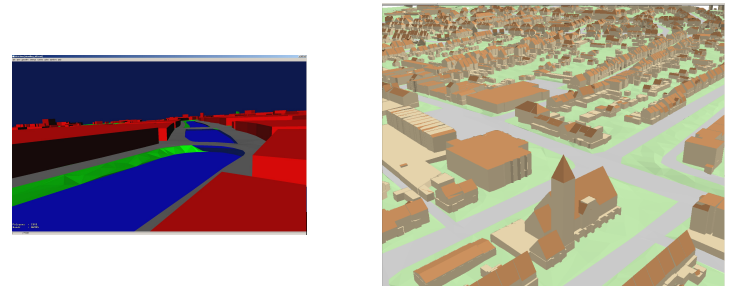

Fig. 8 3D TOP10NL data

Several challenges need further attention before 3D TOP10NL can be put in production. These are:

1. How to reconstruct the data for the whole of the Netherlands given the huge amount of laserdata? How to upscale to larger areas? Generate per partition and glue afterwards?

2. How to maintain and update the 3D data: in a database environment or regenerate complete areas in case of updates? How to assure that $3 \mathrm{D}$ TOP10NL is consistent with $2 \mathrm{D}$ TOP10NL?

3. How to deliver the data? As files (GML? Shape?) or in a Webviewer (WebGL? KML?)? Is it possible to make a dataset of the whole country available at once or should we deliver data sets of specific areas on demand?

\section{3D MAPPING IN SWITZERLAND}

The Swiss Federal Office of Topography (swisstopo) offers a wide range of products ranging from traditional paper maps to digital multimedia products. Since 2008 swisstopo has been developing and updating the Topographic Landscape Model (TLM).

TLM is the basis landscape model for Switzerland. It is a seamless GIS containing 3D primary geometry without generalisation with an accuracy of better than 1meter in $\mathrm{x}, \mathrm{y}$ \& z. TLM consists of 10 themes, which in turn contain several independent datasets previously maintained separately such as boundaries, names and terrain (see Figure 9). It also has a revised extended data model to meet the needs of reference partners such as road and water authorities.

The TLM describes around 10 million natural and artificial landscape features, complete with their position and shape, the corresponding landscape feature type and many other attributes:

- Roads and Tracks (Road and path network, cycling paths and footpaths)

- Public Transportation (Railway tracks and other rail systems, shipping routes and public transport stops) 
- Buildings (Buildings, walls, dams, constructions relating to transport, communication, energy, sport and leisure)

- Areas (Plots with special land usage (transport, habitat, industry etc.))

- Land cover (Ground coverage independent from land usage)

- Hydrography (Watercourses and lake contours)

- Single Point Objects (Selection of prominent landscape features which aid orientation (crossings, sources, caves))

- Names (Various names (mountains, areas, towns))

- Terrain

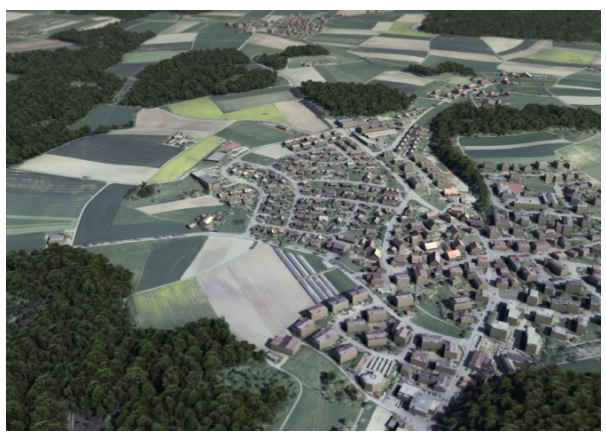

Fig. 9: Representation of swissTLM3D

The content of the TLM and the landscape feature definitions were developed in cooperation with various professional institutions from both the federal government and the cantons. Geometries and a wide range of attributes are provided for all landscape features. All objects have $\mathrm{x}, \mathrm{y}$ and $\mathrm{z}$ coordinates which also means that objects in TLM maintain consistency with DTM-TLM, for example 3D roads should lie on the DTM. TLM is not a product, but rather will be the basis for a wide variety of products including the derived 1:25k cartographic base maps. It serves also the bases for Switzerland's National Spatial Data Infrastructure (NSDI).

\subsection{Production and updating}

The ever-increasing demand for better, more up-to-date diverse digital data has meant that the rapid updating of swisstopo products has taken on a high importance. With the new established production process for TLM an updating infrastructure for a central data set from which all products could be then produced was established.

Reference partners (such as the cadastre, road and rail authorities among others) deliver their up-to-date data for rapid insertion into the TLM in order to maintain top up-to-dateness. The updating process itself is performed in a modern database based system comprising of hardware and software for the capture, editing, management, storage and distribution of the TLM. The associated Digital Terrain Model will also be edited using this infrastructure.

There is one single Corporate Editor enabling staff trained on one part of the production line to easily move to another part of the production line without any technical retraining being required.

\section{Natural and artificial landscape features}

Compared with the formerly existing geodata (VECTOR25, SwissNames, etc.) the TLM offers higher geometric accuracy, but is also more extensive and up-to-date.
The existing data was migrated into the TLM as a basis, mainly due to the rich information in attributes and topology maintained over the years. The data is then updated based on an update cycle of 6 years and improvements and additions are made to the geometries and attributes.

Parallel to the six-year update process, an annual update is carried out through the integration of data from so-called reference partners for selected topics. Initially, this annual update is implemented mainly for the administrative boundaries, buildings and road links. The official cadastral survey plays an important role as a reference partner in this process. The nomenclature for updating names are also integrated from the official cadastral survey.

\section{Terrain}

Existing data is used as a basis: For areas less than $2000 \mathrm{~m}$ above sea level data is used from the laser point survey flights 2000 - 2008. For areas more than $2000 \mathrm{~m}$ above sea level DHM25 was used.

The laser point data is updated in accordance with the TLM update cycle of 6 years (photogrammetric restitution). During this process, the dots are deleted and new points, breaklines and areas are set. For example, incorrect points in areas around bridges are removed and an enclosing breakline (enclosure) is implemented for lakes.

In order to meet the requirements of the TLM with regard to accuracy in areas lying more than $2000 \mathrm{~m}$ above sea level, the DHM25 was replaced in 2010-2012 with more accurate data gained via autocorrelation of aerial photographs. This newly generated data will also be updated in the TLM update cycle.

\subsection{D Products}

Various 3D data sets extracted from the TLM are available since autumn 2010. They comprise the entire territory in reduced form - measured according to the final requirements of the TLM. To be more precise, the number of landscape features on offer is reduced compared with the TLM landscape feature catalogue and the geometric accuracy in the areas where the systematic improvement has not yet been made corresponds to that of data set VECTOR25. This is an initial version (1.0) of the products extracted from the TLM.

The systematic improvement of the data will mean that a Version 2.0 of the various products can be made available alongside Version 1.0. Version 2.0 will be made available sector by sector in accordance with the update blocks. It will contain significantly more landscape features, offer a higher geometric accuracy for all data and will therefore fulfil the requirements with regard to TLM quality.

The TLM is also used as the basis at swisstopo to create the national (2D) maps to a scale of 1:25k, 1:50k and 1:100k.

\section{3D MAPPING AT IGN FRANCE}

\subsection{Early steps}

IGN France did quite a bit of 3D city modelling from the late 70 s until the early $90 \mathrm{~s}$ with the TRAPU product line (Egeles, 1989), based on an in house developed analytic stereoplotting and 3D (actually 2.5D) data management system. Several city LOD2 models were produced in this early period (Figure 10).

This was however a quite expensive product in such times, limited to main city centres, not maintained over time, difficult to use with available GISs of the 80 s, and finally discontinued when a new nationwide product was launched. 


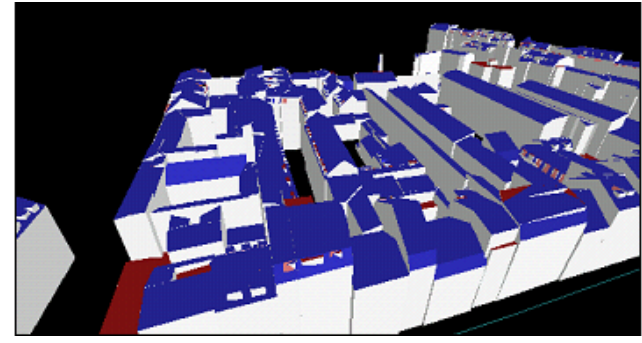

Fig 10 - A TRAPU city model

\subsection{A first nationwide coverage}

The next step, which marked the discontinuation of TRAPU was the BD Topo product, launched in 1994 (Figure 11). This product provides a LOD1 model of buildings as part of a $2.5 \mathrm{D}$ geographic information framework, the Référentiel à grande échelle (RGE, for large scale reference framework). The first French department to be covered was the Vaucluse in 1994, while the nationwide coverage was completed in 2007 only.

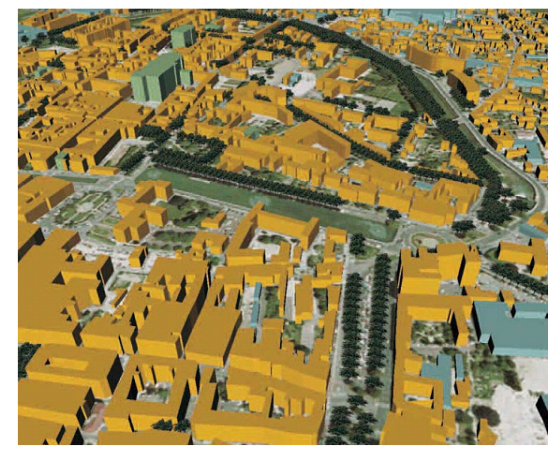

Fig 11 - A view of BD Topo

Each feature comes with a $Z \min$ and $Z$ max value, the $Z$ min value for a building being taken at the gutter level. The BD Topo provides a description for over 40 millions buildings across the country.

Several issues with usage of such data were assessed early on (e.g., see Certu 1999; 2002): data volume and heavy load on computers, limited functionalities of GIS software packages to properly handle $3 \mathrm{D}$ data, staff training, but also content issues: buildings as topographical features do not always match requirements (which may include an interest in ownership divisions, so that BD Topo buildings are progressively merged with cadastral buildings since a few years), visualization of coloured boxes is not always sufficient for communication etc.

An interesting development which followed in the past years is a co-edited product, in partnership with an SME (Archivideo, recently acquired by Dassault Systèmes): Archivideo reprocessed BD Topo buildings to add synthetic textures to roofs and facades, based on the geographic area and building characteristics (Figure 12).

With the addition of the BD Ortho IGN orthoimagery wrapped onto the national DTM, and of 3D synthetic vegetation, such a product provides a much more attractive and realistic visualisation of a geographical area, at low additional cost. It is therefore increasingly used for impact studies, in the first place by local authorities.

This development is interesting on at least two grounds: Firstly because it shows that intermediate, but not 'fully real' products can meet quite a range of requirements, short of more elaborated and complex 3D products secondly, this example shows that tools available on the user side are maturing, including client/server architectures, so as to allow a growth of $3 \mathrm{D}$ data usage.

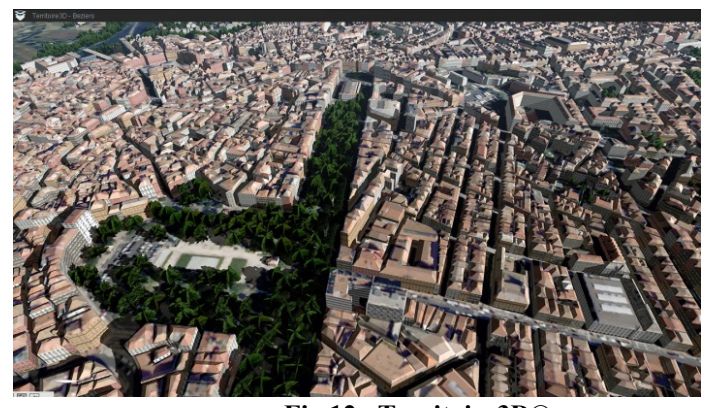

Fig 12- Territoire3D@

\subsection{Closer to full 3D}

In parallel with the completion of the RGE and BD Topo national coverage, IGN launched in late 2007 an innovation project which consisted in industrializing research results pertaining to $3 \mathrm{D}$ data automated extraction, and chose from the beginning to primarily rely upon CityGML and a pivot format in this venture.

This innovation project was completed by the end of 2009, and resulted in au automated workflow currently used for two production activities:

- Creation of high resolution DTMs from dedicated aerial coverages in rural areas ( $25 \mathrm{~cm}$ ground pixel size, typically 4,000 to $10,000 \mathrm{~km}^{2}$ )

- Creation of 3D city models (LOD 2 level) with texturation extracted from images, from higher resolution and denser aerial coverages: that is to say the Bati3D product (Figure 13)

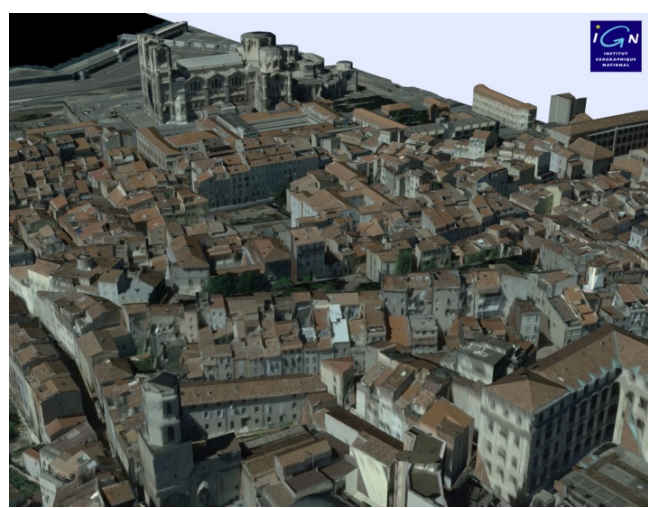

Fig 13 - Marseille old downtown Bati3D

Bati3D is much cheaper to produce than most pre-existing 3D products, in that the bulk of the cost lies in the dedicated aerial coverage, thanks to the research results which were input into the production system (automated building and tree 3D capture). However two lessons seem to emerge:

- Once again issues with the software packages available to users, particularly in terms of data load (as for a big city such as Marseille with a large number of buildings, true textures are a challenge for many visualisation packages, this was exemplified in many instances, including the OGC 3DPIE 2011 testbed, see OGC 2012b)

- Some usage requirements are not met with such a product, either because automated process failures (wrongly depicted roofs) are not acceptable, or because the intended 
usage entails a need for much higher modelling of specific areas (e.g., a new streetcar project impact study will induce a need for a finer modelling of buildings in the considered streets, both in terms of 3D modelling of features that appear in these street and in terms of images used to texture the facades)

This activity led to a partnership with another SME, a both technological and editorial partnership:

- Co-development of software components, starting with a generic 3D I/O library which can cope with most 3D data formats (CityGML, 3DS, KML, Collada), with IGN in charge of CityGML I/O

- Development, here again, of a co-edited enhanced product: the Carto3D city model line, characterised by some level of additional editing and, in the first place, a texturation of facades which is based on images captured by a mobile mapping vehicle (Figure 14)

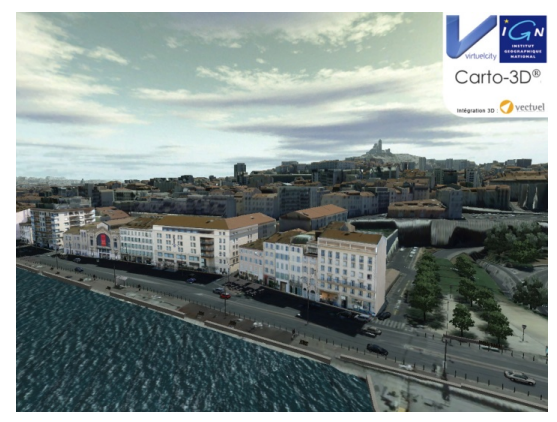

Fig 14 - Marseille old downtown Caro3D

It turns out to be that this later product better meets the requirements of users, but still mostly requirements mostly focused on visualisation of urban areas (in the example above, the whole city of Marseille is available as Bati3D data, while a more limited area in the city centre is provided as Carto3D data).

\subsection{Pending issues as for true 3D data models}

As mentioned above, automated processes are good at creating the bulk of a city model, but failures are costly to identify, and corrective editing can be painstakingly long. Further work on automated identification of failures, and on assisted editing tools are therefore underway, to provide better quality models at an affordable cost.

Compression of textures is another issue, since texture atlases can represent a too heavy load when loading a CityGML dataset, this may be linked with both limitation of the chosen encoding, and of available tools on the user side.

Last but not least, comes the issue of 3D data model maintenance: How to appropriately update such data sets at an affordable cost? There is a whole range of $R \& D$ matters to consider, from aerial coverage options (including UAVs or ultralight aviation) to exploiting images captured from the ground by light cameras (e.g., smartphone cameras). As long as $3 \mathrm{D}$ data is used only for short term communication projects, data maintenance is not an issue, but will turn to be crucial when more elaborated usages develop.

\subsection{CityGML and IGN-F 3D data products}

Insofar CityGML has proved to be up to the task, robust enough to provide a good basis for 3D data exchanges. Only ongoing research suggests that further developments are needed. This being said, many users still require data to be provided in a different data model, because of limited support on the side of software vendors. IGN-F will keep supporting this standard, and contributing to its evolution, in the context of the anticipated development of LOD2 (or higher) data production at the national level.

\section{CONCLUSIONS AND FURTHER WORK}

This paper presents the approaches of national 3D mapping in five states and countries. These approaches show that $3 \mathrm{D}$ mapping activities are no longer limited to project-based, ad hoc and prototype applications. Instead $3 \mathrm{D}$ is considered as an important aspect of existing topographic information workflows.

\subsection{Conclusions}

From the approaches described in this paper, several conclusions can be drawn.

Firstly, 3D data is more and more available but still suffers from a low level of usage (mainly visualisation). In addition standards and formats based on CityGML have been stabilised although software support is still in the early stage. We also observe low level of European harmonisation. A crossEuropean profile for CityGML would be beneficial for several reasons. It would raise the level of adoption of CityGML 3D data, and hence raise usage level; it would push software developers to provide better support for the standard; it would be an important step towards European-wide harmonized data and finally, a better support of the standard would lower the costs needed for data conversion.

Another conclusions that can be drawn from the approaches described in this paper, is that for 3D mapping at the national level, two approaches can be distinguished.

The first approach is followed by ICC, IGN and swisstopo and implements 3D mapping at the most fundamental level. That is, the $3 \mathrm{D}$ data is considered as the prime source of data and $2 \mathrm{D}$ products and services are implemented as derivation of the 3D data. This approach is most sustainable for the future.

The second approach (still) considers the 2D data as prime source of data and extends these data into the third dimension to obtain 3D products and services. This approach is followed by The Netherlands and OSGB. The advantages of this approach are that the transition from $2 \mathrm{D}$ to $3 \mathrm{D}$ is less drastic, the consistency between $2 \mathrm{D}$ and $3 \mathrm{D}$ is assured and that the semantics acquired in 2D can serve as input for automatic reconstruction procedures. The disadvantage is that $3 \mathrm{D}$ information is derived from $2 \mathrm{D}$ and therefore the $3 \mathrm{D}$ mapping is highly driven by $2 \mathrm{D}$ traditions and thinking. In addition, the derivation process of $3 \mathrm{D}$ from $2 \mathrm{D}$ is much more complex than deriving $2 \mathrm{D}$ from $3 \mathrm{D}$ as in the first approach.

Another important conclusion is that, despite maturing 3D technologies, still these are not fully exploited in practice. This has several reasons.

Firstly, 3D is outside the expert and knowledge domain of many organizations. The unfamiliarity with $3 \mathrm{D}$ causes $3 \mathrm{D}$ to be considered as something complicated. This limits actual implementations beyond prototypes and project-based initiatives.

Secondly, there is a big question of who will be the users of 3D and, with that, what will be the user requirements and what is the business case for the needed investments. While these are valid questions for an organisation when considering 3D investments, these questions cannot easily be answered. For the 
digitising of maps resulting in 2D databases, the benefits were clear: initially production lines were improved and later the digitised point, lines and areas could be upgraded to $2 \mathrm{D}$ information needed in spatial analyses. In contrast, 3D does not contribute to the improvement of an existing process. Instead $3 \mathrm{D}$ makes new applications possible and therefore it is much easier to identify the costs to acquire 3D than the benefits of having 3D information available. Many benefits will only become clear after having the $3 \mathrm{D}$ information available for large areas.

Finally, a reluctance to make the step towards 3D mapping can be explained by the lack of a widely adopted 3D standard. While CityGML is a good candidate, software support is limited. In addition, more agreements need to be made at international level on how to implement the standard in specific applications or domains. The 3D profile of the INSPIRE Data Specifications for Buildings, which is based on CityGML, is a good example. We need more of such implementation profiles to offer a solid investment foundation for both organisations and the market.

\subsection{Further work}

Now 3D techniques have matured and OGC has established a standard for 3D data which is well stabilised, in many countries it is no longer a question if countrywide 3D mapping should be realised, but "when" and "how".

From the findings in this paper several issues can be identified that require further (research) attention to progress in the domain of 3D (no ranking):

- More insights in real customer needs of 3D data sets are required (i.e. what topics are relevant and to what extent). These needs should be formulated in terms of what problems 3D can solve. This will stimulate other use of 3D data than visualisation.

- More research is needed on how to fuse data, combining existing data into 3D databases can create problems, i.e. integrating existing non-3D datasets (addresses, boundaries, etc.) with 3D datasets.

- Consistency between 2D and 3D needs further attention. For most mapping authorities 3D geo-information originates from their legacy datasets in $2 \mathrm{D}$. In using this as a basis many associated issues emerge.

- Maintenance and incremental updating of sustainable 3D datasets (sustainability vs. visualization) requires further attention.

- The need of national and European policies on spatial information to stimulate the use of available 3D technologies.

For CityGML, the following recommendations can be formulated for future directions.

Firstly, CityGML needs further support in software systems. This includes implementation of standard products and development of open source tools to support operations. In addition, as a generic standard CityGML needs further agreements defined in profiles. Therefore European CityGML profiles (as the INSPIRE Building profile) need to be defined to harmonise 3D needs and standardise 3D implementations at international level. Relationships with INSPIRE portfolio beyond buildings need to be investigated.

Finally, interfaces with other standards are needed to exchange 3D data with other domains. For example the link with BIMIFC (at least in practical uses) needs to be further investigated.

\section{REFERENCES}

Becker T., C. Nagel, T.H. Kolbe (2010). Integrated 3D modeling of multi-utility networks and their interdependencies for critical infrastructure analysis, 5th International 3D GeoInfo Conference, November 2010, Berlin

Becker, Thomas, Claus Nagel, and Thomas H. Kolbe (2013). "Semantic 3D Modeling of Multi-Utility Networks in Cities for Analysis and 3D Visualization." Progress and New Trends in 3D Geoinformation Sciences. Springer Berlin Heidelberg, 2013. 41-62.

Certu 1999, Evaluation BD Topo ${ }^{\circledR}$ - Synthèse - CERTU, La 3e dimension géographique - Utilisation des modèles numériques de terrain, illustrée par la BD Alti® de l'IGN

Certu, 2002, Evaluation BD Topo $\AA$, online: http://lara.inist.fr/bitstream/handle/2332/1061/CERTU_bdtopo. pdf $\% 3$ Fsequence $\% 3 \mathrm{D} 4$

CityGML, 2013, Application Domain Extensions, [online] www.citygmlwiki.org/index.php/CityGML-ADEs

Czerwinski, A., T. Kolbe, L. Plümer, E. Stöcker-Meier (2006). Interoperability and accuracy requirements for EU environmental noise mapping, proceedings, InterCarto, InterGIS, Berlin 2006.

Czerwinski, A., Sandmann, S., Stšcker-Meier, E. and Pluemer, L. (2007). "Sustainable SDI for EU noise mapping in NRW best practice for INSPIRE." International Journal of Spatial Data Infrastructures Research 2: 90-111.

Geonovum, 2013, EuroSDR/OGC Workshop CityGML in National Mapping,

http://www.geonovum.nl/content/programme-workshopnational-mapping

Geonovum 2012, www.geonovum.nl/3d/toolkit

Egels, Y. (1989) TRAPU, un outil de saisie et de visualisation $\mathrm{du}$ tissu urbain. Metropolis, pages 21-27 http://archives.cerma.archi.fr/sig/pdf/paparoditis.pdf

iScope, 2013, http://www.iscopeproject.net/iscopeNew/

OGC (2012). OpenGIS ${ }^{\circledR}$ City Geography Markup Language (CityGML) Encoding Standard, version 2.0, www.opengeospatial.org/standards/citygml

OGC (2012b) OGC 3D Portrayal Interoperability Experiment Final Report, 27/08/2012

Oude Elberink, Sander, Stoter, Jantien, Ledoux, Hugo, Commandeur, Tom, 2013, Generation and Dissemination of a National Virtual 3D City and Landscape Model for the Netherlands Photogrammetric engineering and remote sensing $\quad 79 \quad 2 \quad 147-158 \quad 2013 \quad$ American Society for Photogrammetry and Remote Sensing

Oude Elberink, S.J. (2010) Acquisition of 3D topography : automated $3 \mathrm{D}$ road and building reconstruction using airborne laser scanner data and topographic maps. Enschede, University of Twente Faculty of Geo-Information and Earth Observation (ITC), 2010. ITC Dissertation 167, ISBN: 978-90-6164-288-6 
ISPRS Annals of the Photogrammetry, Remote Sensing and Spatial Information Sciences, Volume II-2/W1, ISPRS 8th 3DGeolnfo Conference \& WG II/2 Workshop, 27 - 29 November 2013, Istanbul, Turkey

Stoter, J.E., Vosselman, G, Goos, J, S. Zlatanova, E. Verbree, R.Klooster, M. Reuvers, 2011, Towards a National 3D Spatial Data Infrastructure: Case of The Netherlands. - PFG Photogrammetrie, Fernerkundung, Geoinformation 2011(6): 405-420.

Stoter, J.E., L. van den brink, J. Beetz, H. Ledoux, M. Reuvers, R. Klooster, P. Janssen, F. Penninga, G. Vosselman, 2013, Establishing and implementing a national 3D standard, Journal for Photogrammetry and Geoinformation (PFG), In press

Van den Brink, L., Jantien Stoter2, Sisi Zlatanova, 2013, UMLBased Approach to Developing a CityGML Application Domain Extension, Transactions in GIS, Article first published online: 22 May 2013 DOI: 10.1111/tgis. 12026

Van Den Brink, Linda, Stoter, Jantien en Zlatanova, Sisi, 2012, Establishing a national standard for 3D topographic data compliant to CityGML, in: International Journal of Geographical Information Science, in press.

URL:

http://www.tandfonline.com/doi/abs/10.1080/13658816.2012.66 7105 